\title{
CAPÍTULO 03: FATORES CLIMÁTICOS E A INCIDÊNCIA DA DENGUE NO MUNICÍPIO DE PICOS-PI
}

\section{CAPÍTULO 03: FACTORES CLIMÁTICOS Y LA INCIDENCIA DEL DENGUE EN EL MUNICIPIO DE PICOS-PI}

\section{CHAPTER 03: CLIMATE FACTORS AND THE INCIDENCE OF DENGUE IN THE MUNICIPALITY OF PICOS-PI}

\author{
Italo Giullian Carvalho de Albuquerque ${ }^{1}$; José Anatiel Gonçalves Santos Landim²
}

DOI: https://doi.org/10.31692/978-65-88970-06-5.30-47

\begin{abstract}
RESUMO
A dengue é um problema de saúde pública e para combatê-la, é necessário conhecer cada vez mais os fatores favoráveis ao seu desenvolvimento, proliferação, modos de transmissão e outras informações inerentes. Estudos realizados demonstram que existe relação entre as variáveis climáticas e o aumento do número de casos de dengue. Diante disso, o presente trabalho buscou analisar as relações entre as variáveis climáticas com os casos notificados de dengue no município de Picos-PI no período entre 2015 e 2019. Foram utilizados dados de casos de dengue e do clima em Picos, disponibilizados pelas bases de dados do DATASUS e INMET. A pesquisa tem caráter descritivo e é qualitativa. Utiliza ainda uma análise exploratória de dados referentes à dengue no município em questão. Foram feitas análises utilizando as bibliotecas da linguagem de programação Python, e as bibliotecas pandas e matplotlib, onde foi possível fazer a manipulação e a visualização da aplicação do algoritmo. Como resultado, verificou-se que os casos de dengue estão relacionados regularmente com as mudanças climáticas na cidade de Picos-PI, tendo maior incidência no período entre março e junho, quando as condições climáticas são favoráveis: aumento de chuva e da umidade relativa do ar, e a diminuição da temperatura. Na referida cidade estudo revela um elevado índice de notificação de dengue nos meses frios e úmidos, sugerindo políticas públicas de prevenção, bem como a conscientização da população nas ações disciplinares para essa época do ano. Como o clima é mais quente na segunda metade do ano, registra-se um baixo número de casos significante da dengue.
\end{abstract}

Palavras-Chave: Dengue, Clima, Dados.

\section{RESUMEN}

El dengue es un problema de salud pública y para combatirlo es necesario conocer cada vez más los factores favorables a su desarrollo, proliferación, modos de transmisión y demás información inherente. Los estudios han demostrado que existe una relación entre las variables climáticas y el aumento del número de casos de dengue. Ante esto, el presente estudio buscó analizar la relación entre las variables climáticas con los casos reportados de dengue en el municipio de Picos-PI en el período entre 2015 y 2019. Se utilizaron datos sobre casos de dengue y clima en Picos, puestos a disposición por las bases de datos. datos de DATASUS e INMET. La investigación es descriptiva y cualitativa. También utiliza un análisis exploratorio de datos relacionados con el dengue en el municipio en cuestión. Los análisis se realizaron utilizando las bibliotecas del lenguaje de programación Python y las bibliotecas pandas y matplotlib, donde fue posible manipular y visualizar la aplicación del algoritmo. Como resultado, se encontró que los casos de dengue se relacionan regularmente con el cambio climático en la ciudad de Picos-PI, con mayor incidencia en el período comprendido entre marzo y junio, cuando las condiciones climáticas son favorables: aumento de las precipitaciones y humedad relativa en la ciudad. aire, y la disminución de la temperatura. En esa ciudad, un estudio revela una alta tasa de notificación de dengue en los meses fríos y húmedos, sugiriendo políticas

1 Bacharelado em Ciência da Computação, Instituto de Educação italoalbuq@hotmail.com

Especialista em Engenharia de Sistemas, Instituto de Educação Superior Raimundo anatielsantos@gmail.com

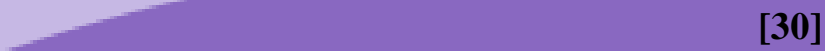


públicas de prevención, así como la concienciación de la población sobre las acciones disciplinarias para esa época del año. Como el clima es más cálido en la segunda mitad del año, hay un número significativamente bajo de casos de dengue.

Palabras Clave: Dengue, Clima, Datos.

\begin{abstract}
Dengue is a public health problem and to combat it, it is necessary to know more and more the factors favorable to its development, proliferation, modes of transmission and other inherent information. Studies have shown that there is a relationship between climatic variables and the increase in the number of dengue cases. In view of this, the present study sought to analyze the relationship between climatic variables and the reported cases of dengue in the municipality of Picos-PI in the period between 2015 and 2019. Data on dengue cases and the climate in Picos, made available by the databases, were used. data from DATASUS and INMET. The research is descriptive and qualitative. It also uses an exploratory analysis of data related to dengue in the municipality in question. Analyzes were made using the Python programming language libraries, and the pandas and matplotlib libraries, where it was possible to manipulate and visualize the application of the algorithm. As a result, it was found that dengue cases are regularly related to climate change in the city of Picos-PI, with a greater incidence in the period between March and June, when climatic conditions are favorable: increased rainfall and relative humidity in the city. air, and the decrease in temperature. In that city, a study reveals a high dengue notification rate in the cold and humid months, suggesting public prevention policies, as well as the population's awareness of disciplinary actions for that time of year. As the climate is warmer in the second half of the year, there is a significant low number of dengue cases.
\end{abstract}

Keywords: Dengue, Climate, Data.

\title{
INTRODUÇÃO
}

Segundo a Organização Mundial de Saúde (OMS), mais da metade da população mundial vive sob riscos de transmissões ou em áreas endêmicas da doença dengue, e isso a torna uma das principais questões da saúde pública no mundo. Os países mais atacados pela doença são os tropicais e subtropicais, nos quais o ambiente climático favorece o ciclo de vida do transmissor (BRASIL, 2015).

A dengue tem como seu principal vetor o mosquito Aedes aegypt, é constituída por quatro sorotipos: DEN-1, DEN-2, DEN-3, DEN-4 e é caracterizada por ser febril aguda.O meio de transmissão é pela difusão da picada do mosquito. A emergência da infecção sob formas de Dengue Clássica, Síndrome de Choque da Dengue e Febre Hemorrágica da Dengue coloca essa virose como um dos casos mais graves de saúde pública das Américas (SECRETARIA DA SAÚDE DO PARANÁ, 2020).

Está presente em 4.318 municípios brasileiros (77\% das cidades brasileiras), tendo seu primeiro caso em 1982, na cidade de Boa Vista, Roraima. Nessa época, o Brasil atingiu uma elevada endemia, em virtude do rápido contato do vetor em um grande alargamento territorial, o que assegurou a circulação do vírus em grandes números de estados e munícios, expondo aos poucos novas populações a infecções (SOUSA; DANTAS; LIMEIRA, 2007).

A contingência da dengue no Brasil e no mundo está relativamente associada à urbanização sem estruturas de saneamento. Este fator contribui com a aglomeração ativa do 
mosquito e também a disseminação dos vários sorotipos da doença. Neste campo de interação entre meio ambiente e saúde, as variáveis climáticas como umidade relativa do ar, temperatura e precipitação pluviométrica podem ser estudadas para verificar sua associação a doença da dengue.

Neste contexto, esta pesquisa tem como objetivo analisar as relações entre as variáveis climáticas com os casos notificados de dengue no município de Picos-PI no período entre 2015 e 2019, visando o aprimoramento da vigilância e controle da doença no município. Para isso, foram identificados a disseminação de casos da dengue no município de Picos-PI, as variáveis meteorológicas no referido período, e a relação que elas tem em comum.

\section{FUNDAMENTAÇÃO TEÓRICA}

A dengue é um vírus de genoma RNA (Ácido ribonucleico) e a doença é transmitida pelo mosquito vetor fêmea Aedes aegypti, da família Flaviviridae. Apresenta quatro sorotipos conhecidos (DEN-1, DEN-2, DEN-3, DEN-4). É uma doença infecciosa que pode causar desde pequenas infecções assintomáticas e febres constitucionais até formas mais graves que pode ocasionar a morte, como manifestações hemorrágicas. É considerada a arbovirose mais importante que afeta ao homem e é um problema de saúde mundial. Sua convivência com o ser humano é favorecida pelo uso de recipientes artificiais no seu desenvolvimento, condição que torna o vírus predominantemente urbano (SECRETARIA DA SAÚDE DO PARANÁ, 2020).

O primeiro caso de dengue no Brasil, aconteceu no ano de 1982, na cidade de Boa Vista, Roraima, mas, somente em 1986 foi conceituada como epidemia explosiva e atingiu todas as regiões brasileiras. Nessa ocasião, o país alcançou um alto índice endêmico, pois, o vetor se dispersou de forma rápida em grande extensão territorial. Isso ocasionou uma circulação do vírus em maior número de estados e municípios, exibindo novas populações às infecções (SOUSA; DANTAS; LIMEIRA, 2007).

Conforme o relato do Ministério da Saúde (2015), através do Plano de Contingência Nacional para Epidemias da Dengue, no Brasil o padrão epidemiológico vai se alterando conforme o passar dos anos. Primordialmente, era comum os casos de dengue acontecer em pessoas adultas e jovens. Entre 2007 e 2009 foi alterado esse resultado, aumentando as formas graves principalmente em crianças.

No Brasil, a dengue se manifesta de modo sazonal, ocorrendo na maioria dos casos entre os meses de outubro e maio, sendo menos frequente entre dezembro e março. Este período é perceptível na maioria dos estados. A incidência sobe significativamente nos 
primeiros meses do ano, alcançando maior destaque de março a maio, seguida de uma redução considerável dos casos a partir de junho.

Pena (2020), retrata que o clima é o resultado de toda espécie de fenômenos meteorológicos específicos, que definem a situação média da atmosfera, em uma determinada região da superfície terrestre. As variações climáticas interferem diretamente no processo saúde-doença, provocando impactos positivos e negativos na qualidade de vida e de saúde das populações.

Os fatores climáticos são estudados, para que possam ser deduzido hipóteses de causalidade quanto a algum fator de risco cuja permutação na natureza dependa da variação de algum fator climático. (MINISTÉRIO DA SAÚDE, 2020). O que mais tem influência nas comunidades biológicas são os aspectos climáticos: umidade relativa do ar, temperatura e precipitação pluviométrica.

As epidemias da dengue estão relacionadas com as flutuações climáticas, pois se tratam de arbovirose (doenças causadas pelo vírus da dengue, zika vírus, febre chinkungunya e febre amarela), e os aspectos climáticos estão associados a sobrevivência e reprodução de vetores. Moore (1985), identificando a grandeza de relações entre Aedes aegypti e a fatos climáticos, apresentou que a temperatura não foi uma boa referência de abundância larval, porém o volume e o número de dias com chuva podem constituir preditores úteis de sua abundância. Segundo Reiter (1988), isso geralmente é válido, todavia, deve-se considerar que as taxas de infecção viral no vetor também variam com as condições climáticas.

A temperatura ambiente tem ligação com o tamanho da população, período de maturação, atividade de alimento com sangue da fêmea Aedes aegypti e a taxa de sobrevivência do vetor da dengue. A temperatura abala o comportamento do vírus no vetor. A maturação e replicação do vírus no inseto são rápidas com o aumento da temperatura, aumentando assim, a analogia de vetores no ambiente e sua aplicação na transmissão do vírus, ampliando a magnitude das epidemias (HALES; WET; MAINDONALD; WOODWARD, 2002).

De maneira contrária, a temperatura ambiente não é proporcional ao tempo de aumento do Aedes aegypti para a fase adulta, porém, acima de $40^{\circ} \mathrm{C}$ a expectativa de vida do mosquito amortece. As taxas máximas de sobrevivência estão presente na faixa de $20^{\circ}$ a $30^{\circ} \mathrm{C}$, no entanto, o vetor sobrevive acima $\operatorname{dos} 30^{\circ} \mathrm{C}$ com um mínimo de impacto (PATZ; MARTENS; FOCKS; JETTEN, 1998).

Segundo Yasuno e Tonn (1990), as temperaturas máximas, mínimas e médias durante o dia foram testadas como preditivos para disseminação sazonal de dengue, e a temperatura 
mínima mostrou ser o melhor pressagiador. As temperaturas mínimas levam a decadência da taxa de repasto sanguíneo, abaixando a taxa de contato entre mosquitos e humanos, o que pode atingir a taxa de transmissão viral. Elas aparentam ser críticas para a continuidade do mosquito e a propagação da população vetorial.

A temperatura mínima limiar para a pertinência do vírus da dengue foi considerada em $11.9^{\circ} \mathrm{C}$, e o vírus não é ampliado no vetor quando a temperatura está inferior a $18^{\circ} \mathrm{C}$. Em temperaturas muito baixas, o crescimento viral é mais lerdo e o mosquito não sobrevive tempo bastante pra se tornar infeccioso e transmitir o vírus (HALES; WET; MAINDONALD; WOODWARD, 2002).

A umidade relativa do ar é a combinação da quantidade de água existente no ar e da quantidade máxima que poderia conter na mesma temperatura. Simplificando, ela é o total de água em forma de vapor existente na atmosfera no momento, em relação ao total máximo que poderia existir na temperatura analisada. Ela é um dos indicadores usados na meteorologia para saber como o tempo se comportará (fazer previsões). A umidade do ar é mais baixa no final do inverno e início da primavera (CENTRO DE GERENCIAMENTO E DE EMERGÊNCIAS CLIMÁTICAS, 2020).

De acordo com o InfoEscola (2006), dependendo da temperatura, ocorre uma variância na umidade relativa do ar. A $0^{\circ} \mathrm{C}$, a umidade relativa do ar é de $4,9 \mathrm{~g} / \mathrm{m}^{3}$, e a $20^{\circ} \mathrm{C}$ é de $17,3 \mathrm{~g} / \mathrm{m}^{3}$. Varia também de acordo com a presença ou não de florestas ou vegetação, rios e represas, e à queda da temperatura.

A umidade relativa atinge a existência do vetor, tanto na fase de ovo como na forma adulta. A concordância de umidade e calor toma força com a junção de hábitos alimentares e atração entre os mosquitos (LIANG; HUALIAN; WEIZHONG; JIMIN; QIYONG, 2009). Na época em que a umidade no ambiente é relativamente baixa, pode beneficiar a fêmea do mosquito na procura de humanos para alimentação, proporcionando a transmissão da doença.

O índice pluviométrico é o resultado do somatório da quantidade da precipitação de água em um determinado local durante um período de tempo. Existem três diferentes causas da formação de chuva, e todas tem a ver com a ascensão de massa de ar quente e úmida na atmosfera.

A primeira causa é a Precipitação Orográfica, que ocorre quando uma massa de ar quente e úmida se move ao longo de uma região.

A segunda é a Precipitação Convectiva, que resulta como uma massa de ar instável e se eleva na atmosfera a partir de uma área que se aqueceu.

A última é a Precipitação Frontal que resulta do confronto entre duas grandes massas 
de ar, uma massa quente e outra massa fria. Se a massa fria é a que avança, o resultado é uma frente fria; se a quente avança, uma frente quente se desenvolve (OLIVEIRA, 2020).

O aumento da precipitação ocasionaria o acréscimo no número de locais para a reprodução das larvas do vetor da dengue, devido ao acúmulo de água. Todavia, chuva em excesso poderia eliminar o habitat por causa das enchentes, o que resultaria a diminuição da população vetorial. (PATZ; MARTENS; FOCKS; JETTEN, 1998). Níveis de chuva muito baixos leva o acúmulo de água em reservatórios que podem também ser geradores. Nesse caso, a precipitação poderá ou não ser relacionada com a doença conforme as características locais.

A Ciência de Dados inclui vários elementos e baseia-se em teorias e técnicas derivadas de vários campos da engenharia e da ciência. A Ciência de Dados surge como ingrediente cada vez mais importante em várias áreas, tais como saúde, financeira, astronomia, bioinformática, segurança digital e entre outros (PORTO; ZIVANI, 2014). Ela permite aplicar técnicas com finalidade de descoberta de padrões como também de descoberta de conhecimento através de bases de dados.

Pode-se enumerar os passos para o processo de descoberta correspondendo às etapas deste processo, que são: seleção, pré-processamento, transformação, mineração, interpretação e avaliação.

Figura 01: Processo de descoberta de conhecimento em base de dados.

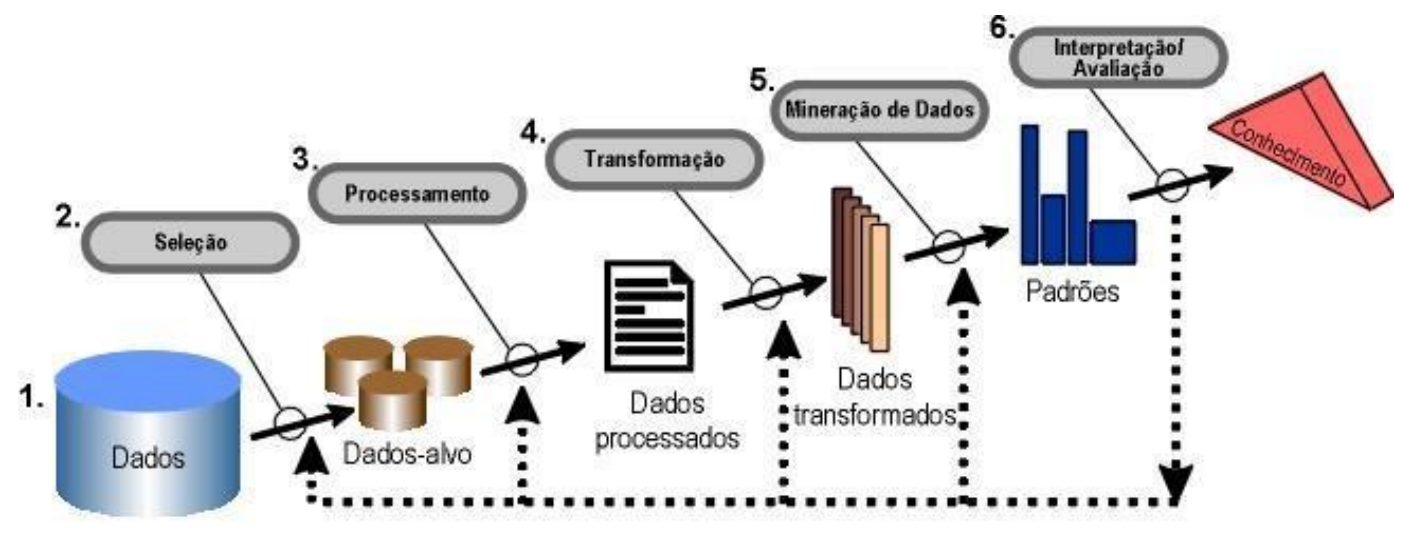

Fonte: Loureiro, et al. (2014).

1 - Compreender o domínio do conhecimento importante e dos objetivos do usuário.

2 - Criar o conjunto de dados para a utilização através da seleção dos dados ou atributos relevantes (seleção).

3 - Pré-processar os dados, limpeza e remoção de ruído e desvios, estratégia de correção de dados desconhecidos ou faltantes, normalização, remoção de atributos 
numéricos de documentos e logs (pré-processamento).

4 - Reduzir e projetar novamente os dados, isto pode ser atuado através da seleção de atributos relevantes para representar melhor os dados sem perda de precisão, constantemente dependendo do objetivo a ser atingido (transformação).

5 - Definir o método de mineração considerando o objetivo do processo (classificação, regressão, agrupamento).

6 - Estabelecer os algoritmos de mineração de dados com base no objetivo geral e na estrutura imposta aos dados. A definição dos algoritmos envolve a escolha de modelos, parâmetros e formas de execução.

7 - Minerar os dados em busca de amostras de interesse usando algoritmos e dados selecionados (mineração).

8 - Interpretar os resultados e avaliar os padrões e regras achados pelo processo de mineração. (interpretação).

9 - Avaliar os conhecimentos adquiridos, documentação e elaboração de relatórios (avaliação) (NETO, 2019).

A análise das características presentes em um dado conjunto de dados, possibilita o descobrimento de padrões e tendências comportamentais que podem apresentar informações sobre os processos que os gerou. Muitas informações podem ser obtidas por simples estatísticas, outras através de técnicas de visualização de dados (HAN; KAMBER, 2011).

O pré-processamento de dados é um conjunto de técnicas de mineração de dados utilizadas para transformar dados brutos em modelos úteis e eficientes. Existem três principais passos que envolvem este processo: limpeza de dados, transformação de dados e redução de dados. A limpeza de dados se enquadra com o manuseio ou preenchimento de dados ausentes, redução de ruídos, identificação e remoção de valores aberrantes e a resolução de inconsistências. A transformação de dados é realizada para transformar os dados originais em modelos mais apropriados e adequados para o processo de mineração. E a redução de dados é usada para enfrentar o grande volume de dados (DATA GEEKS, 2020).

Este conjunto de técnicas se torna um processo semiautomático, pois entende-se que essa fase necessita da capacidade do analista de dados em identificar os problemas que estão presentes nos dados, como também a natureza desses problemas com o objetivo de utilizar os métodos mais apropriados para solucionar cada um dos problemas (BATISTA, 2003).

Batista (2003), ainda destaca que, na fase de normalização dos dados, os valores dos atributos e variáveis são desfigurados de seus intervalos originais para um intervalo específico. Essas transformações são especialmente valiosas para métodos que calculam 
distâncias entre variáveis.

O Python é uma linguagem de programação interpretada, de script, funcional, orientada a objetos, imperativa, de alto nível, de tipagem forte e com semântica dinâmica. É uma das linguagens que mais cresce, devido sua compatibilidade capacidade de auxiliar outras linguagens, e também a linguagem mais utilizada para análise de dados (CAELUM, 2020).

Inicialmente, era comporta por uma comunidade relativamente pequena, de engenheiros e cientistas que usavam o Python para necessidades de computação. Migrou para a utilização do módulo numérico, na qual tratava do desenvolvimento de operações simbólicas que envolviam fatoração de derivadas, integrais e polinômios, como também cálculo de matrizes (MILLMAN; AIVAZIS, 2011). Esta comunidade continuou com o avanço do módulo numérico do Python e começou a desenvolver e compartilhar os pacotes adicionais para a computação científica.

Hoje, códigos científicos requer não somente números, desempenho ou facilidade de uso, mas geralmente precisam de protocolos de rede de suporte, aplicativos baseados na Web, aplicativos baseados em banco de dados, e gráficos sofisticados com interfaces. Esta visão geral argumenta que o Python aumentou com uma pilha de ferramentas desenvolvido especificamente para computação científica e forma um ambiente altamente produtivo para a computação moderna (MILLMAN; AIVAZIS, 2011).

Além dos usuários individuais, o Python é aplicado também em produtos de empresas reais, como o Google e o Yahoo! que o utiliza em serviços de internet, a IBM que o usa para testes de hardware, a Light and Magic que utiliza o Python para produção de animação, entre outras (LUTZ; ASCHER, 2007).

O uso do Python para análise de dados, exploratória e visualização de dados é impreterivelmente comparada com as muitas outras linguagens de programação, como $\mathrm{R}$ e MATLAB. Essa linguagem ganhou destaque tanto em programação de propósito geral quanto como uma linguagem única para criação de aplicações focadas em computação científica e análise de dados (MATOS, 2020).

\section{METODOLOGIA}

Para realização desse trabalho, recorre-se a abordagem quantitativa de pesquisa, na qual é "à linguagem matemática para descrever as causas de um fenômeno, as relações entre variáveis, etc." (FONSECA, 2002, p. 20). Esse tipo de abordagem se concentra na 
objetividade, uma vez que considera que a realidade há de ser compreendida com base na análise de dados. Também aborda a qualitativa, que segundo Pádua (2017), objetivam da densidade às análises dos dados coletados, evidenciando toda a subjetivação do conteúdo para que possa gerar uma discussão crítica e reflexiva sobre o assunto.

O trabalho compreende uma pesquisa descritiva, que segundo Gil (2016, p. 42), "têm como objetivo primordial a descrição das características de determinada população ou fenômeno ou, então, o estabelecimento de relações entre variáveis". Essa abordagem vai além do simples reconhecimento da existência de afinidade entre variáveis, e pretendem ajustar a natureza dessa relação. Também faz parte de um outro tipo, a pesquisa exploratória, que objetiva proporcionar maior coleguismo com o problema, com concepções a torná-lo mais coerente ou a constituir hipóteses (GIL, 2016).

Neste estudo se faz necessário o uso de uma metodologia que consiste em pesquisas utilizando-se referências bibliográficas detalhadas e o levantamento das controversos da dengue. Com esse levantamento, a pesquisa busca detectar relações entre a incidência de casos de dengue e as variáveis climáticas no município de Picos-PI no período entre 2015 e 2019.

O seio desta pesquisa é analisar os dados referente às notificações de casos de dengue, como também dados climatológicos na cidade de Picos-PI.Para a captura dos dados, foi usado como base, os órgãos públicos que são responsáveis por esse armazenamento dos dados e sua divulgação.

Pelo DATASUS (Departamento de Informática do Sistema Único de Saúde do Brasil), através dos boletins epidemiológicos emitidos pela Secretaria de Vigilância em Saúde, a fim de monitorar e investigar doenças específicas sazonais no Brasil, foi possível utilizar os dados de casos notificados de dengue para a tabulação no dataset criado. O DATASUS é alimentado pela investigação de casos de doenças e agravos constantes da lista nacional de doenças de notificação compulsória. A entrada de dados é feita a partir de instrumentos de coleta padronizados, como a Ficha Individual de Notificação (FIN) e a Ficha Individual/de Investigação (FII) (BRASIL, 2008).

Os dados referentes ao clima foram obtidos através do INMET (Instituto Nacional de Meteorologia), por meio do BDMEP (Banco de Dados Meteorológicos para Ensino e Pesquisa). Foi possível selecionar diversas variáveis climáticas no período referido. O INMET provém de informações meteorológicas alcançada por meio de monitoramento, análise e previsão de tempo e de clima, que se fundamentam em pesquisa aplicada, trabalho em parceria e, compartilhamento do conhecimento, com ênfase em resultados práticos e $[38]$ 
confiáveis. O sistema de coleta e distribuição de dados meteorológicos do instituto é dotado de estações de sondagem de ar superior (radiossonda), estações meteorológicas de superfície, e a maior rede de estações automáticas da América do Sul (INMET, 2020).

Os dados foram tratados em conjunto com a etapa de criação da base de dados utilizada, verificando a presença de atributos e valores nulos, como também a formatação dos valores presentes nos dados capturados. Dessa maneira, os dados puderam ser avaliados de maneira inicial, evitando erros de processamento na etapa de análise. As variações climáticas mensais, foram avaliadas por estudo direto e por métodos estatísticos.

Após a seleção das bases de dados utilizadas, os dados foram tabulados e organizados em uma planilha eletrônica contendo 61 linhas referentes aos meses de cada ano e 8 colunas referentes às variáveis analisadas.

Em seguida, os dados foram convertidos para o formato CSV (Valores Separados por Vírgula), pois com esse formato é possível fazer importação e exportação para planilhas e bancos de dados usados em Python, como mostra a Tabela 01 os valores iniciais do arquivo.

Tabela 01: Amostra dos dados utilizados

\begin{tabular}{rrrrrrrr}
\multicolumn{2}{c}{$\begin{array}{c}\text { Evapotranspiracao } \\
\text { Data }\end{array}$} & Insolacao & Precipitacao & TemperaturaMaxima & Temperaturaminima & UmidadeRelativa & CasosDengue \\
\hline $\mathbf{2 0 1 5 - 0 1 - 3 1}$ & 46.600000 & 257.9375 & 46.600000 & 35.98500 & 23.80500 & 50.25000 & 15.0 \\
$\mathbf{2 0 1 5 - 0 2 - 2 8}$ & 91.300000 & 162.6000 & 84.300000 & 34.37800 & 23.59600 & 64.82100 & 13.0 \\
$2015-03-31$ & 54.354052 & 257.9375 & 47.611765 & 35.42586 & 23.07798 & 58.28304 & 64.0 \\
$2015-04-30$ & 74.124000 & 268.1000 & 41.300000 & 33.73600 & 22.35300 & 67.56600 & 205.0 \\
$2015-05-31$ & 32.549000 & 242.7000 & 19.700000 & 34.37700 & 21.95400 & 56.25800 & 97.0
\end{tabular}

Fonte: O autor (2020).

A Tabela 2 descreve os tipos de dados referentes as variáveis utilizadas, a fim de especificar melhor para a análise posteriormente.

Tabela 02: Tipos de variáveis presentes

\begin{tabular}{|c|c|}
\hline VARIÁVEL & UNIDADE DE MEDIDA \\
\hline Data & data (dia/mês/ano) \\
\hline Evapotranspiração & $\mathrm{mm}$ (milímetros) \\
\hline Insolação & hs (horas) \\
\hline Precipitação & $\mathrm{mm}$ (milímetros) \\
\hline TemperaturaMáxima & ${ }^{\circ} \mathrm{C}$ (graus celsius) \\
\hline TemperaturaMínima & ${ }^{\circ} \mathrm{C}$ (graus celsius) \\
\hline UmidadeRelativa & $\%$ (porcentagem) \\
\hline CasosDengue & inteiro \\
\hline \multicolumn{2}{|c}{} \\
\hline
\end{tabular}


Por fim, as análises foram feitas com o auxílio da linguagem de programação Python, junto com algumas bibliotecas, como: pandas e matplotlib.

O município de Picos, está localizado na região centro-sul do Estado do Piauí, situado na Latitude Sul de $7^{\circ} 05^{\prime} 13^{\prime \prime}$ e Longitude Oeste de 41 28' 12", posicionada a 202 metros de altitude, com uma população estimada de 78.222 habitantes, possui área total de $534,7 \mathrm{~km}^{2}$. É a maior produtora de mel do país, ficando em $1^{\circ}$ lugar no ranking nacional (IBGE, 2020). O bioma é a caatinga e apresenta o clima o semiárido, caracterizando uma temperatura média anual de $26.5^{\circ} \mathrm{C}$, com baixa amplitude térmica anual da temperatura média.

Em Picos, a estação com imponderação é quente, abafada e de céu encoberto; a estação seca é muito quente, de ventos fortes e de céu parcialmente encoberto. Ao longo do ano, a temperatura varia de $21^{\circ} \mathrm{C}$ a $39^{\circ} \mathrm{C}$ e raramente é inferior a $20^{\circ} \mathrm{C}$ ou superior a $40^{\circ} \mathrm{C}$. A estação quente é entre setembro e novembro, com temperatura máxima média diária acima de $37^{\circ} \mathrm{C}$. A estação fresca permanece de janeiro a abril, com temperatura máxima diária em média abaixo de $33^{\circ} \mathrm{C}$ (WEATHER SPARK, 2020).

A estação de maior precipitação dura quatro meses, que compreende de dezembro a abril, com probabilidade acima de $27 \%$ de que um determinado dia tenha chuva. Já estação seca dura oito meses, de abril a dezembro. O período chuvoso do ano dura sete meses, de outubro a maio, com precipitação de chuva de 31 dias contínuos, mínima de 13 milímetros. O máximo de chuva ocorre durante os 31 dias ao redor de 16 de março, com acumulação total média de 134 milímetros. O período sem chuva do ano dura cinco, que compreende maio a outubro. O mínimo de chuva ocorre por volta de 17 de agosto, com acumulação total média de 0 milímetro (WEATHER SPARK, 2020).

\section{RESULTADOS E DISCUSSÃO}

Segundo levantamento do período de epidemia da dengue em Picos-PI, foi apurado que, o surto na cidade, geralmente se dá entre os meses de março a junho, e com isso, não segue o mesmo padrão epidemiológico do restante do Brasil, uma vez que o período epidemiológico no Brasil ocorre entre outubro a maio. Essas informações podem ser analisadas a partir das figuras a seguir: 
Figura 02: Comportamento da dengue nos anos de 2015 a 2019 no município de Picos-PI.
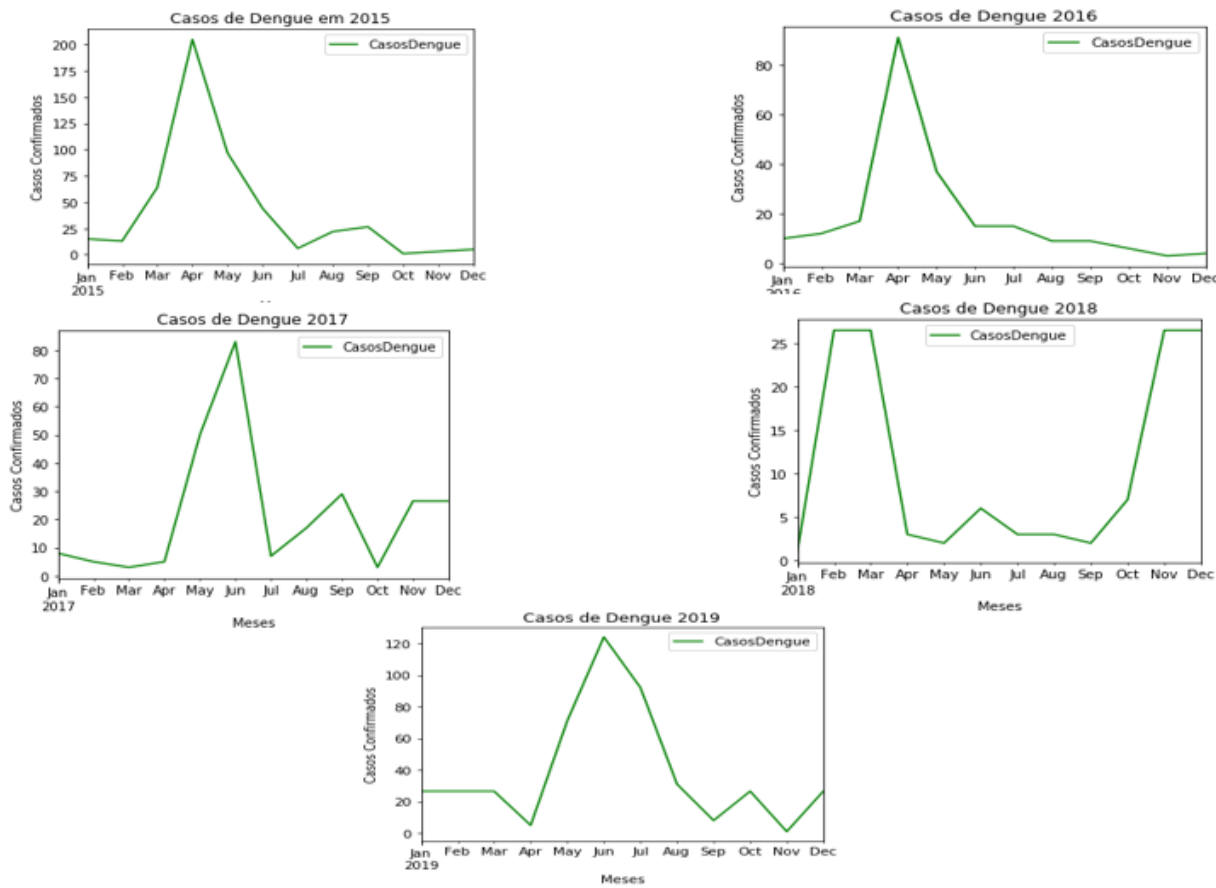

Fonte: O autor (2020).

Em 2018, os níveis apresentados de casos são baixos ou quase nulos, demonstrando que a ocorrência da dengue neste ano não apresentou um comportamento capaz de ser previsto através de uma análise inicial dos dados. Essas características podem estar relacionadas a campanhas governamentais e medidas preventivas adotadas pela população, de modo que não se observou um grande surto neste ano. A imagem anterior, mostra que o período de dengue apresenta um surto frequente entre os anos, destacando-se que os gráficos estão de acordo com a média de cada ano.

A Figura 03, apresenta o comportamento da dengue em um comparativo entre todos os anos analisados, 2015 a 2019.

Figura 03: Comportamento da dengue, em média, nos anos de 2015 a 2019 no município de Picos-PI.

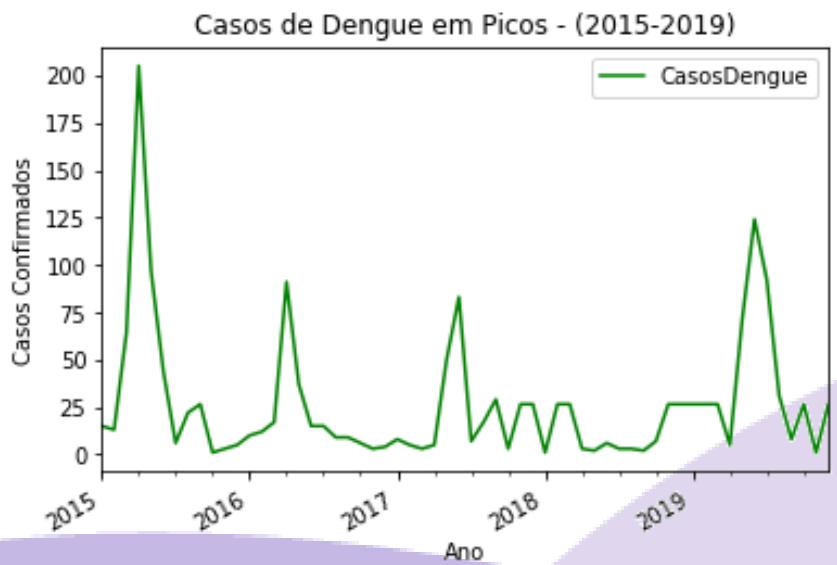

Fonte: O autor (2020). 
Os dados apresentados permitem verificar a curva da incidência da dengue nos anos correspondentes entre 2015 e 2019, onde pode-se concluir que 2015 foi o ano disparado que obteve mais casos e 2019 em sequência.

A Figura 04 abaixo, apresenta a variação dos dados em relação às chuvas durante os meses de cada ano, demonstrando que existe um aumento das chuvas no início do ano e diminuição a partir da segunda metade do ano. No período em 2018, houve uma quantidade considerável na queda das chuvas, isso induz as baixas notificações significativas de casos de dengue no município nesse período.

Figura 04: Comportamento das chuvas (mm), nos anos de 2015 a 2019 no município de Picos-PI.

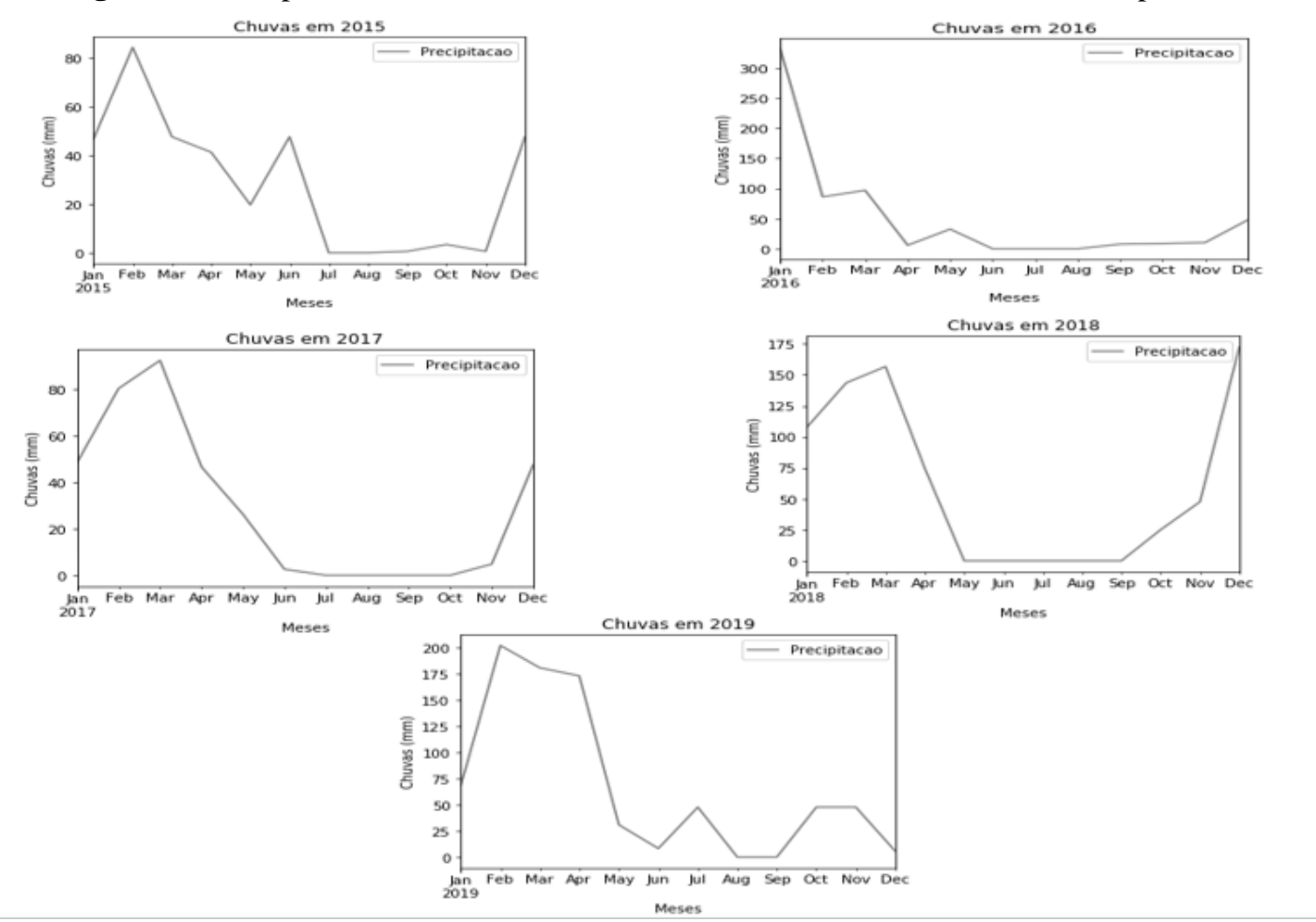

Fonte: O autor (2020).

O período correspondente entre maio e outubro apresenta poucas chuvas, e este período é o de menor incidência da dengue na cidade. Esse agente não é observado em outros estados do Brasil onde a estação de grandes incidências da dengue combina com o período chuvoso, segundo o Ministério da Saúde (FARHAT, 2003).

A umidade relativa do ar tem relação direta com a propagação da dengue, estando diretamente ligada aos padrões da doença. Na Figura 05, é constatado a variação média durante o período de estudo, apresentando de maneira também semelhante ao padrão de distribuição do comportamento da dengue. 
Figura 05: Relação da dengue coma umidade relativa do ar (\%), nos anos de 2015 a 2019 no município de Picos-PI.

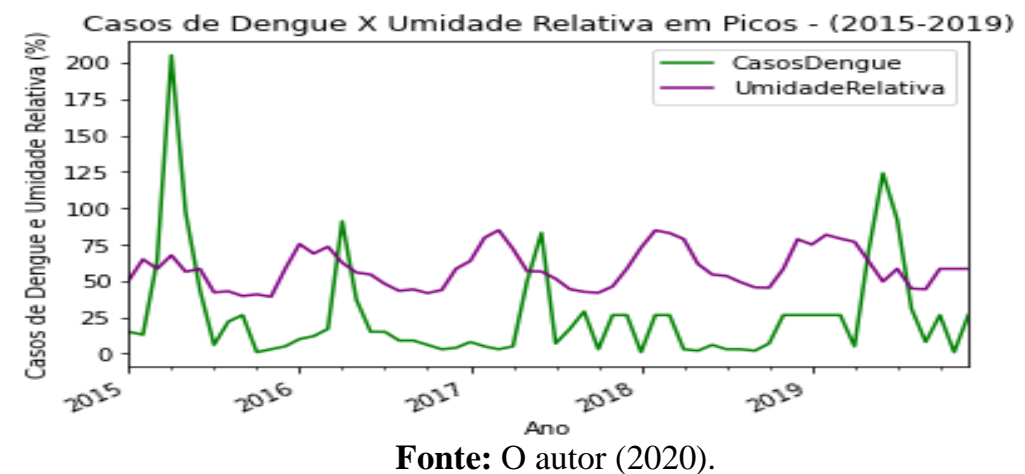

Outro fator que atenua a propagação da dengue é a temperatura. Na Figura 06, vê-se a variação média da temperatura máxima no decorrer de todos os anos analisados e a variação média da temperatura mínima no mesmo período. É possível notar que a medida em que passa os meses, a temperatura mínima e máxima decai no primeiro semestre, e voltam a subir a partir do segundo semestre do ano. Esta relação é inversa à da umidade, que tem seu aumento na primeira parte do ano e decréscimo a partir do segundo semestre. Logo, conforme a temperatura abaixa, a umidade relativa do ar aumenta.

Figura 06: Temperaturas máximas e mínimas $\left({ }^{\circ} \mathrm{C}\right)$, nos anos de 2015 a 2019 no município de Picos-PI.
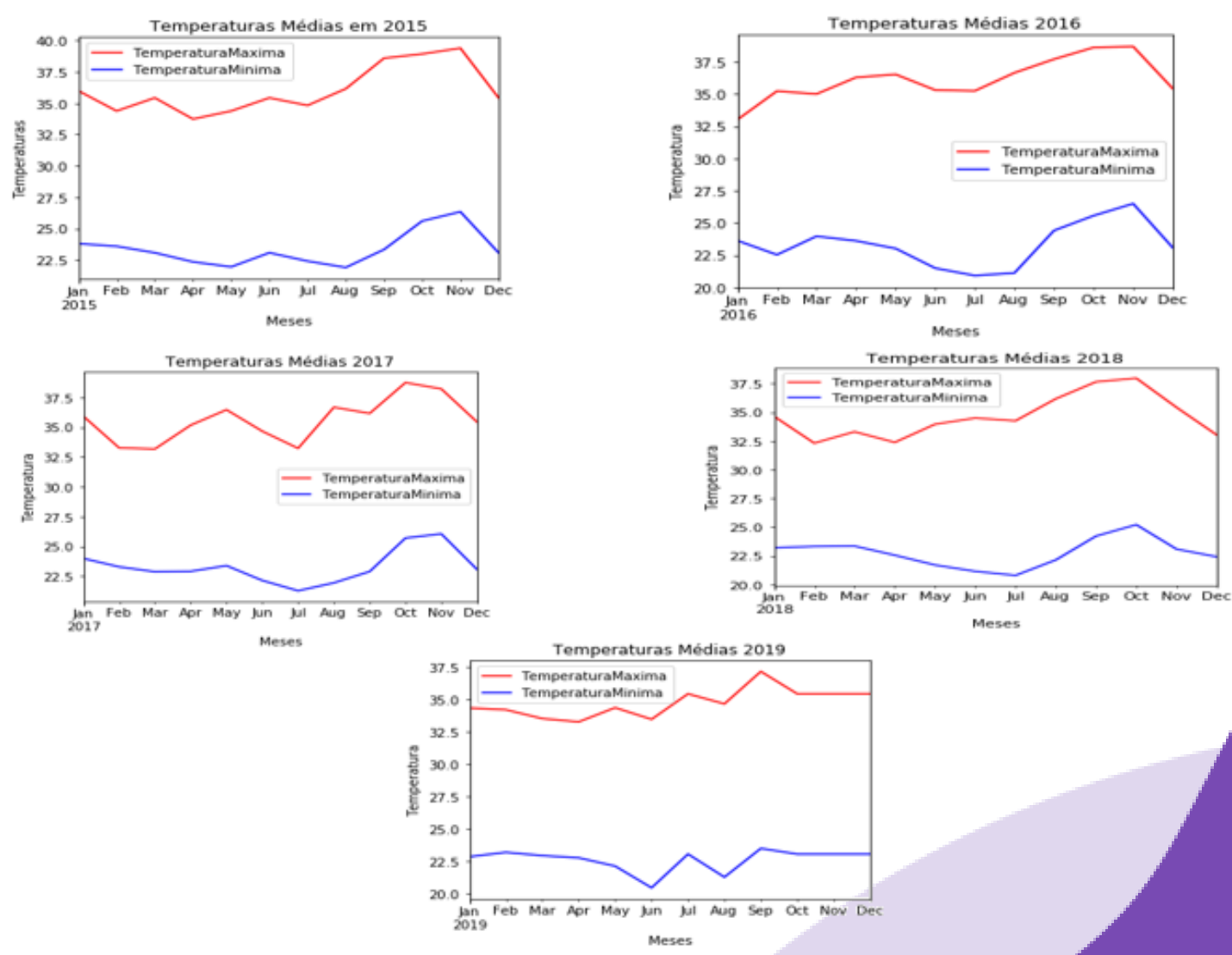

Fonte: O autor (2020). 
É visto, através da imagem anterior, que a dengue possui um comportamento relativo durante o primeiro semestre do ano, em que os casos aumentam na condição da diminuição da temperatura, aumento da chuva e aumento da umidade.

\section{CONCLUSÕES}

Através dessa pesquisa, foi possível perceber que a dengue possui padrões comportamentais complexos no município de Picos-PI, tornando o reconhecimento de relações entre as variáveis climatológicas e os casos de dengue difícil. A análise de dados mostra que a dengue apresenta um comportamento distinto na cidade de Picos, pois os fatores climatológicos demonstraram dados específicos do município, visto que as mudanças climáticas variam de maneira diferente do restante do Brasil.

Verificou-se que, os casos de dengue estão relacionados regularmente com as variações da temperatura, precipitação e umidade relativa do ar, tendo como fatores principais a alta da chuva, a alta da umidade relativa do ar, e, a queda da temperatura durante o ano, permitindo concluir que o período de maior propagação da dengue é entre maio e junho. Aguarda-se uma melhor compreensão que possa ajudar a entender e antecipar processos complexos e indiretos. A mudança global do clima, políticas públicas de combate à dengue e, medidas de prevenção da população se tornam eficientes nesse momento.

De modo geral, as mudanças climáticas de precipitação, umidade relativa do ar e temperatura podem influenciar o comportamento e a distribuição dos vetores transmissor, alterando assim, a incidência de doenças transmitidas por vetores. Ressalta-se também que, não se pode admitir que o clima seja fator único que afete a transmissão da dengue. Todavia, ainda existe grandes diferenças fundamentais entre uma abordagem baseada em dados e uma abordagem baseada em cenário.

Por fim, fica esclarecido que mesmo sendo uma doença que está fortemente relacionada às mudanças climáticas, a dengue pode ser influenciada por diversos outros aspectos, como fatores ambientais, sociais e populacionais. As políticas públicas de saúde para o combate e erradicação da doença podem influenciar na maneira como os padrões epidemiológicos se comportam, tendo como foco os programas e atividades de conscientização da população, modificando o comportamento dos dados dos casos de dengue no município de Picos.

\section{O dataset e os códigos utilizados podem}




\section{REFERÊNCIAS}

BATISTA, Gustavo Enrique de Almeida Prado Alves. Pré-processamento de dados em aprendizado de máquina supervisionado. 2003. 231 f. Tese (Doutorado) - Curso de Instituto de Ciências Matemáticas e de Computação, Instituto de Ciências Matemáticas e de Computação, São Carlos, 2003.

BRASIL. Ministério da Saúde. Secretaria de Vigilância em Saúde. Departamento de Vigilância das Doenças Transmissíveis. Plano de Contingência Nacional para Epidemias de Dengue / Ministério da Saúde, Secretaria de Vigilância em Saúde, Departamento de Vigilância das Doenças Transmissíveis. - Brasília: Ministério da Saúde, 2015. 42 p.

BRASIL. MINISTÉRIO DA SAÚDE. VIGILÂNCIA EM SAÚDE: Dengue, Esquistossomose, Hanseníase, Malária, Tracoma e Tuberculose. 2. ed. Brasília: Ministério da Saúde, 2008. 119 p.

Condições meteorológicas médias de Picos Brasil. Weather Spark, 2020. Disponível em: $<$ https://pt.weatherspark.com/y/30826/Clima-caracter\%C3\%ADstico-em-Picos-Brasildurante-o-ano>. Acesso em 17 de jun. de 2020.

FARHAT, R. Dengue: Brasil prepare-se para a batalha. InformeSaúde, v. 7, n. 196, 2003.

FONSECA, J. J. S. Metodologia da pesquisa científica. Fortaleza: UEC, 2002.

GIL, Antônio Carlos. Como elaborar projeto de pesquisa. São Paulo: Atlas, 2016.

GOMES, Pedro Cesar Tebaldi. Conheça as etapas do pré-processamento de dados. Datageeks, 2019. Disponível em: <https://www.datageeks.com.br/pre-processamento-dedados/>. Acesso dia 23 de maio de 2020.

HAN, Jiawei; KAMBER, Micheline. Data Mining: Concepts and Techniques. 2. ed. São Francisco: Elsevier, 2006.

INSTITUTO BRASILEIRO DE GEOGRAFIA E ESTATÍSTICA (IBGE). Cidades: Picos, Piauí, 2018. Disponível em <https://cidades.ibge.gov.br/brasil/pi/picos/panorama>. Acesso em: 17 mai. 2020.

INSTITUTO NACIONAL DE METEOROLOGIA (INMET). Precipitação Nacional de Meteorologia, 2020. Disponível em < http://www.inmet.gov.br/portal/>. Acesso em: 18 jun. 2020.

J.A. Patz, WJ Martens, D.A. Focks, and T.H. Jetten. Dengue fever epidemic potential as projected by general circulation models of global climate change. Environmental Health Perspectives, 106(3):147, 1998.

L. Liang, L. Hualiang, T. Linwei, Y. Weizhong, S. Jimin, and L. Qiyong. Time series analysis of dengue fever and weather in Guangzhou, China. BMC Public Health, 9, 2009.

LOUREIRO et al. Indução de árvores de decisão na descoberta de conhecimento: caso de 
empresa de organização de eventos. IV Congresso Internacional de Casos Docentes de Marketing Público e Não Lucrativo, 2014. Disponível em: $<$ https://www.researchgate.net/figure/Figura-2-Processo-de-descoberta-de-conhecimento-embases-de-dados_fig1_270449012>. Acesso dia: 10 de jun. de 2020.

LUTZ, Mark; ASCHER, David. Aprendendo Python. 2 ed, Porto Alegre: Bookman, 2007.

MATOS, David. R ou Python para Análise de Dados? Ciência de dados, 2020. Disponível em:< http://www.cienciaedados.com/r-ou-python-para-analise-de-dados/>. Acesso em: 29 de maio de 2020.

MELLOR, P.S.; LEAKE, C.J. Climatic and geographic influences on arboviral infections and vectors. Revue Scientifique Et Technique de L'oie, [s.1.], v. 19, n. 1, p.41-54, 1 abr. 2000. O.I.E (World Organisation for Animal Health). http://dx.doi.org/10.20506/rst.19.1.1211.

MILLMAN, K. Jarrod; AIVAZIS, Michael. Python for Scientists and Engineers. Computing In Science \& Engineering, [s.1.], v. 13, n. 2, p.9-12, mar. 2011. Institute of Electrical and Electronics Engineers (IEEE). http://dx.doi.org/10.1109/mcse.2011.36.

Mudanças Climáticas. Ministério de Saúde, 2020. Disponível em: $<$ https://www.saude.gov.br/vigilancia-em-saude/vigilanciaambiental/vigidesastres/mudancasclimaticas>. Acesso dia 21 de maio de 2020.

NETO, José Trajano Mendes. Utilização da ciências de dados para identificação da relação entre fatores climáticos e a incidência de casos de dengue no município de PatosPB. Trabalho de conclusão de curso (graduação) - Universidade Estadual da Paraíba, Patos PB, 2019.

OLIVEIRA, Aureo S. de. Precipitação Pluviométrica. In: OLIVEIRA, Aureo S. de. Fundamentos de Meteorologia e Climatologia. NEAS/UFRB. Cap. XI. Disponível em: <www.ufrb.edu.br > 8-cca-035-meteorologia-e-climatologia-agricola >. Acesso en $20 \mathrm{de}$ maio de 2020.

O que é Python. CAELUM, 2020. Disponível em: < https://www.caelum.com.br/apostila-pythonorientacao-objetos/o-que-e-python/\#null>. Acesso em: 29 de maio de 2020.

PÁDUA, Elisabete Matallo Marchesini de. Metodologia da pesquisa: abordagem teóricoprática. 20 ed. Campinas, SP: Papirus, 2017. 97 p. (Coleção Magistério: formação e trabalho pedagógico).

PORTO, F. A. M; ZIVIANI, A. Ciência de Dados. In: 3o. Seminário de Grandes Desafios da Computação no Brasil, 2014, Rio de Janeiro, RJ. Anais do 3o. Seminário de Grandes Desafios da Computação, 2014.

S. Hales, N. de Wet, J. Maindonald, and A. Woodward. Potential effect of population and climate changes on global distribution of dengue fever: an empirical model. The Lancet, 360(9336):830-834, 2002.

SOUSA, Nadja Maria Nascimento; DANTAS, Renílson Targino; LIMEIRA, Rodrigo Cézar. Influência de Variáveis Meteorológicas Sobre a Incidência Do Dengue, Meningite e Pneumonia em João Pessoa-Pb. Revista Brasileira de Meteorologia, 2007. São José dos 
Campos - SP, v.22, n.2, p. 183-192, agosto de 2007.

Umidade relativa do ar. Centro de Gerenciamento de Empresas Climáticas da Prefeitura de São Paulo. Disponível em: 〈https://www.cgesp.org/v3/umidade-relativa-do-ar.jsp>. Acesso em: 21 de maio de 2020.

(Vigilância Ambiental - Vetores) Informações Básicas - Dengue. Secretaria da Saúde do Paraná, 2020. Disponível em:

<http://www.saude.pr.gov.br/modules/conteudo/conteudo.php?conteudo=370>. Acesso em: 8 de mar. 2020. 